Disponível em:

http://editora.unoesc.edu.br/index.php/race

RACE, Unoesc, v. 14, n. 3, p. 905-924, set./dez. 2015

\title{
EFEITOS DA URGÊNCIA SOBRE O NÍVEL DE PARTICIPAÇÃO EM PROCESSOS DELIBERATIVOS
}

Effects of the urgency on the participation level in deliberative processes

Leonardo Secchi

E-mail: leosecchi@gmail.com Pós-doutor em Políticas Públicas pela Universidade de Wisconsin (EUA); Doutor em Estudos Políticos pela Universidade de Milão (Itália); Professor de Administração Pública na Universidade do Estado de Santa Catarina; Membro-diretor da

Sociedade Brasileira de Administração. Endereço para contato: Avenida Madre Benvenuta, 2037, Itacorubi, 88035001, Florianópolis, Santa Catarina, Brasil.

Juliana Kulpa Feijó E-mail: juli2809@gmail.com Graduada em Administração Pública pela Universidade do Estado de Santa Catarina.

Letícia Elena Ito E-mail: leticia.naturologa@gmail.com Graduanda em Administração Pública pela Universidade do Estado de Santa Catarina.

Artigo recebido em 10 de fevereiro de 2015. Aceito em 17 de agosto de 2015. 
Resumo

Neste trabalho se apresentam os resultados obtidos a partir da análise da influência da urgência sobre o nível de participação em contextos deliberativos. A hipótese básica nesta pesquisa é que quanto menor o tempo disponível para deliberar mais concentrado é o processo deliberativo (menor nível de participação). Para testar a hipótese, foi realizado um estudo quase-experimental, no qual indivíduos foram submetidos a simulações de deliberação com alta, média e baixa urgências para posterior análise sobre o impacto da variável independente (urgência) sobre a variável dependente (nível de participação). As simulações foram gravadas em áudio e vídeo e, posteriormente, analisadas quanto à participação de cada indivíduo em relação ao total de participação dos grupos. Para a mensuração do nível de participação, adaptou-se a metodologia de cálculo do Coeficiente de Gini, adaptado para mensurar a concentração/distribuição da quantidade de intervenções dos sujeitos de pesquisa durante o processo deliberativo. Os resultados indicam que a urgência tem influência negativa sobre o nível de participação em contextos deliberativos, corroborando a hipótese da pesquisa.

Palavras-chave: Deliberação. Participação. Urgência. Tomada de decisão.

\section{Effects of the urgency on the participation level in deliberative processes}

\section{Abstract}

In this paper we present the results from a research that analyzed the influence of urgency on the level of participation in deliberative contexts. The basic hypothesis on this research is that the shorter the time available to deliberate the more concentrated is the deliberative process (less participation). To test the hypothesis, we conducted a quasi-experimental study, in which two groups of people simulated deliberations with high, medium and low urgency, in order to assess the impact of the independent variable (urgency) upon the dependent variable (level of participation). The simulations were audio and video recorded, and, later, analysed according to each participant's participation in relation to the total of the groups' participation. In order to measure the level of participation, we adapted the Gini coefficient methodology for calculating concentration/distribution of interventions of the participants in the deliberative process. The results indicate that urgency has negative impact on the level of participation in deliberative contexts, supporting the research hypothesis.

Keywords: Deliberation. Participation. Urgency. Decision-making.

\section{INTRODUÇÃO}

A participação é benéfica para o processo de tomada de decisão? Essa simples pergunta provoca disputa de argumentos de equidade, eficiência, eficácia, qualidade e resiliência, que são respondidos de forma diferenciada entre matrizes 
normativas e teóricas das ciências sociais aplicadas, em geral, e da teoria das decisões, em específico.

As teorias não contingenciais de participação asseguram que o envolvimento de indivíduos em um processo decisório independe de aspectos externos e que o julgamento sobre o benefício da participação está fundado em axiomas normativos favoráveis ou desfavoráveis à participação.

As teorias contingenciais, no entanto, propõem que o nível "ideal" de participação depende das condições ambientais e do perfil dos participantes. As teorias contingenciais sobre liderança e participação em processos decisórios (FIEDLER, 1967; GRANDORI, 1984; VROOM; JAGO, 1988; HERSEY; BLANCHARD, 1993; PAPADAKIS; LIOUSKAS; CHAMBERS, 1998) têm mostrado que o nível de participação varia de acordo com fatores ambientais e fatores relativos aos participantes.

Entre os fatores ambientais, pode-se listar a urgência, a tecnicidade, a necessidade de legitimação e a necessidade de sigilo (SIMON, 1977; PAPADAKIS; LIOUSKAS; CHAMBERS, 1998; IRVIN; STANSBURY, 2004; GUNDERSEN; BOYER, 2012). Entre os fatores relativos aos participantes estão habilidade, motivação, necessidade de aprendizado, custos transacionais e custos de oportunidade (COASE, 1960; STEINER, 1972; NAGEL, 1987; BARTOL; MARTIN, 1991; PAYNE; BETTMAN; LUCE, 1996). Em maior ou menor escala, esses fatores afetam o nível de participação em decisões coletivas, em organizações públicas e privadas (SECCHI; PLEBANI, 2006).

Os processos de tomada de decisão coletiva podem ser diferenciados em dois modelos: modelo decisionístico, no qual é feita a soma de preferências individuais (soma de votos individuais) ou modelo deliberativo, em que há aglutinação das preferências individuais (debate e tentativa de consenso) (AVRITZER, 2000).

Para efeitos de operacionalização dos conceitos, a urgência é aqui entendida como pressão ou constrangimento gerado pela escassez de tempo (time pressure). O nivvel de participação é operacionalizado como a distribuição das contribuições argumentativas dos diversos membros presentes em um contexto decisório, sendo alto o nível de participação quando todos se envolvem e baixo quando apenas um indivíduo concentra o processo decisório. Deliberação, ou contexto deliberativo, aqui, é entendido como um debate em que indivíduos falam e escutam sucessivamente buscando alcançar uma decisão coletiva por meio de trocas de razões e argumentos (BOHMAN, 1996; CURINI, 2006). 
Um dos fatores relativos ao contexto foi a urgência e seus reflexos sobre o nível de participação em contexto deliberativo. O objetivo com o estudo que originou a este artigo foi o de analisar os efeitos da urgência sobre o nível de participação em processos deliberativos. O recorte deste artigo traz os resultados da análise empírica desse fator e se a variável independente (urgência) causa alguma influência - se sim, positiva ou negativa - em relação à variável dependente (nível de participação).

A hipótese básica nesta pesquisa é que quanto menor o tempo disponível para deliberar, menor também será o nível de participação dos indivíduos (alta concentração deliberativa nas mãos de poucas pessoas). Para testar essa hipótese, foi empreendido um estudo quase-experimental por intermédio de três simulações com estudantes universitários e três simulações com aposentados, envolvendo alta, média e baixa urgência.

Por meio desse estudo, além disso, buscou-se contribuir para as discussões sobre teorias contingenciais do nível de participação de um processo deliberativo, as quais afirmam que o nível "ideal" de participação depende diretamente das condições do ambiente. Em contraste, as teorias não contingenciais entendem que o grau de participação e o estilo de liderança em nada dependem dos aspectos externos.

A relevância do tema é evidente. Organizações públicas e privadas enfrentam diariamente dilemas relativos ao nível de participação que devem oportunizar a seus membros e clientes/usuários em reuniões, na discussão de problemas e em soluções organizacionais. Portanto, do ponto de vista prático, neste artigo buscou-se trazer indicações para a resolução de trade-offs decisórios sobre o nível de participação.

Do ponto de vista teórico, visou-se contribuir para o avanço das teorias contingenciais de decisão sobre a adequação ou desejabilidade da participação em processos deliberativos. Este estudo filia-se a uma agenda de pesquisa das teorias contingenciais sobre participação e liderança, em que se tem estudado a influência de fatores relativos ao ambiente e a fatores relativos aos participantes sobre a tomada de decisão. Buscou-se, aqui, elucidar se a quantidade de tempo disponível para o processo deliberativo afeta o nível de participação dos sujeitos envolvidos.

\section{PARTICIPAÇÃO, DELIBERAÇÃO E URGÊNCIA}

Segundo Vroom e Jago (1988), a participação é entendida como o resultado da influência de uma pessoa do grupo que assumiu um papel ativo no processo de tomada de decisão. Um dos modelos de participação é o deliberativo, no qual 
pessoas trocam razões e argumentos para tentar chegar a uma decisão consensual. Exemplos de arenas deliberativas são os conselhos de acionistas em empresas, conselhos consultivos e os conselhos gestores na administração pública.

Tem-se por contexto deliberativo um cenário de tomada de decisão coletiva em que os envolvidos tentam chegar a um consenso entre si por meio do intercâmbio de razões e argumentos (CURINI, 2006; SECCHI; PLEBANI, 2006). Baseado no fundamento de Habermas, segundo Barisione (2012), na deliberação é preciso ter espaços de discussão que adotem certas características, como: igualdade entre as pessoas, principalmente no quesito da não existência de hierarquia de poder, e tempo semelhante para cada participante se expressar, objetivando a não influência de seus integrantes ou evitando a utilização do espaço como simples ato simbólico. Em tal processo, o número e a variedade de pessoas inseridas afetam diretamente a forma e o conteúdo da decisão.

Ao analisar o processo deliberativo no contexto de uma decisão governamental, por exemplo, tanto os participantes quanto o governo obtêm ganhos (IRVIN; STANSBURY, 2004). Para os cidadãos, o aprendizado e o ganho de habilidades são notáveis durante o processo; nos resultados o ganho é o controle sobre as decisões. Já o governo ganha maior legitimidade nas decisões, conseguindo construir a confiança da população e evitando os possíveis custos de litígio, além dos ganhos de amplitude de visão para a tomada de decisão.

Contudo, desvantagens tanto aos organizadores do processo deliberativo quanto aos participantes também podem ser citadas. Entre elas, há o custo de tempo para todos e o risco de a decisão ser capturada pelos grupos de interesse presentes nos processos deliberativos. Da mesma forma, quando uma decisão resultante de tal consenso é ignorada, cresce o sentimento de ressentimento e impotência por parte dos participantes, criando contestações sobre a legitimidade do processo.

Para Farrar et al. (2010), a deliberação, frequentemente, traz mais mudanças de atitude e faz com que preferências tenham maior unificação (alteração de preferências individuais), pois grande parte das pessoas tende a aproximar suas posições às de outras que já se manifestaram. O processo deliberativo se distingue do processo de tomada de decisão coletiva (modelo decisionístico) exatamente nesses elementos homegeneizadores dos interesses e entendimento. O princípio da regra e o voto majoritário presentes no modelo decisionístico constrange a participação e a construção coletiva, a favor de uma individualização do julgamento.

Quanto ao nível de participação no processo deliberativo, Secchi e Plebani (2006) propõem algumas hipóteses ao analisar a influência que certas variáveis têm 
na deliberação. Entre as hipóteses está a de que, depois de certo nível, a margem de contribuição de cada participante no processo deliberativo diminui gradativamente à medida que novos membros são incorporados no processo. Também foram feitas hipóteses sobre consensos influenciados pela tecnicidade (decisões programadas e não programadas), pela habilidade (experiência, criatividade e outras capacidades) e pela motivação dos participantes. Secchi e Plebani (2006) também propuseram que as arenas deliberativas possuem uma relação centro-periférica, ou seja, algumas pessoas assumem papéis mais relevantes e de centralidade administrativa, técnica ou decisória, obtendo um status maior que outros participantes do grupo (periferia), existindo um mínimo grau de hierarquia, mesmo que informal.

Entre os fatores que afetam o nível de participação, a urgência é uma das mais citadas (BEN-ZUR; BREZNITZ, 1981; SECCHI; PLEBANI, 2006; GUNDERSEN; BOYER, 2012). O tempo, afirma Elias (1998), é um símbolo que atribui ao homem um guia ao mundo e de regulação da convivência no espaço social. Ainda que exista uma concepção de tempo de cada indivíduo, tanto no sentido de amadurecimento emocional quanto físico, há também o tempo externo em que o ambiente demanda uma reação do indivíduo, influenciando o seu comportamento.

Estudos mais recentes na área de comportamento organizacional apontam que não é apenas a urgência que afeta o processo decisório, mas a percepção individual sobre o seu próprio tempo e o tempo dos outros membros (CALSYDE; KEREN; ZEELENBERG, 2014).

De acordo com Emmendoefer (2004), o tempo pode ser classificado em: duração, sequência, sincronização, periodicidade e ritmo. Para melhor compreensão da urgência, é preciso entender a definição de ritmo. O ritmo, segundo o autor, está relacionado com a velocidade e a intensidade, ou seja, a partir do momento em que há mudança no primeiro, consequentemente, haverá mudança nesses outros fatores. A intensidade está relacionada com atividades, que são uma sequência de eventos. A mudança de intensidade reflete no intervalo de tempo - maior ou menor - entre um evento e outro, relacionando-se à velocidade, aceleração e desaceleração.

A influência da urgência sobre o processo decisório é bastante estudado em razão de suas consequências cognitivas. Ben-Zur e Breznitz (1981) apontam que os três principais efeitos são a aceleração do processo decisório (menor processamento de informações), a maior seletividade das informações e as mudanças nas estratégias de escolha, passando de um modelo em base de alternativas para um modelo mais genérico de escolha em base de atributos (PAYNE; BETTMAN; LUCE; 1996). Para Gundersen e Boyer (2012), os processos deliberativos pressionados pela urgência 
devem ser chamados de just in time exploratory discussion, com diferenças marcantes de processos deliberativos sem restrição de tempo.

A pergunta que permanece é: o nível de participação em um contexto deliberativo é afetado pelo nível de urgência? Os procedimentos metodológicos desta pesquisa foram desenhados para responder essa pergunta.

\section{PROCEDIMENTOS METODOLÓGICOS}

Na pesquisa que originou a este artigo, optou-se por uma abordagem teóricoempírica, de metodologia hipotético-dedutiva e de caráter quase-experimental (KERLINGER, 1980; SELTTIZ; WRIGHTSMAN; COOK, 1987).

Os estudos experimentais, de acordo com Gil (2011), permitem que seja possível, ao escolher o objeto de estudo, distinguir as variáveis que podem influenciá-lo, ter mais controle sobre ele e averiguar quais efeitos a variável causa nele. São estudos que possuem dois grupos, um deles experimental e o outro controle.

Já nas pesquisas quase-experimentais, não é possível atingir um grau de controle equivalente ao do delineamento experimental, podendo ser realizadas com pessoas não equivalentes ou, até mesmo, com as mesmas pessoas em diferentes momentos (GIL, 2011; COZSBY, 2006). Apesar disso, ainda é factível constatar o que acontece, quando acontece e a quem acontece; portanto, é possível averiguar relações de causa-efeito (GIL, 2011).

Os quase-experimentos foram realizados com dois grupos de pessoas, ambos afiliados a uma universidade: uma turma com o total de 24 alunos de um curso de extensão voltado para pessoas da terceira idade (aposentados) e uma turma com o total de 18 alunos do sexto termo de um curso de graduação (alunos). ${ }^{1}$

Como limitação para a realização dos quase-experimentos, houve a dificuldade no recrutamento de pessoas, levando a equipe a articular com turmas de alunos da universidade na qual a pesquisa foi realizada e tornando o critério de seleção dos grupos não aleatório. Tal fato pôde interferir nas deliberações pela convivência e amizade ou conhecimento que os participantes já tinham uns com os outros, pois nessas situações é possível que algumas pessoas queiram evitar o confronto de opiniões com as outras em nome do relacionamento que possuem fora da arena deliberativa. Contudo, os experimentos foram realizados de forma não esperada pelos participantes, evitando que combinações prévias existissem. 
Os quase-experimentos foram realizados em salas de aula disponibilizadas pela universidade onde foi realizada a pesquisa. O layout das salas de aula dos quaseexperimentos foi formado por cadeiras dispostas em um grande semicírculo (formato ferradura) para facilitar a visualização e a troca de informações pelos participantes e, também, para viabilizar a captura de imagem de todo o ambiente com a câmera de vídeo. Além desta, a equipe de pesquisa utilizou dois gravadores de áudio para ter mais fontes de captação de som, distribuídas pelo semicírculo.

Para simulação do processo deliberativo, a equipe de pesquisa adaptou três cases de tomada de decisão chamados Abrigo Subterrâneo. Nesses cases é descrita uma situação hipotética de crise nuclear mundial em que a espécie humana corre risco de extinção e a única forma de perpetuação da espécie é proteger algumas pessoas (máximo seis) em um abrigo subterrâneo. O objetivo do grupo era deliberar sobre quais seriam as seis pessoas a serem salvas entre os 12 personagens descritos, os quais variavam entre pessoas drogadas, pessoas com autismo, jovens, idosos e outras pessoas, de ambos os sexos, com características diversas. Esse tipo de dilema envolve questões éticas e valorativas relacionadas ao valor da vida e à utilidade humana, que geram muito debate e argumentação. Além disso, o Abrigo Subterrâneo é uma simulação standard, na qual variam os personagens e suas características em cada case realizado, garantindo que a única variável em tratamento seja a urgência.

Os sujeitos de pesquisa foram colocados em situação de deliberação com três níveis de urgência: baixa urgência, com limitação de 20 minutos para a deliberação, média urgência, com limitação de cinco minutos, e alta urgência, com limitação de dois minutos. Para cada uma das simulações os cases foram idênticos, mas com personagens do Abrigo Subterrâneo diferentes. Os cases foram os mesmos para o grupo de alunos e o grupo de aposentados, mas em momentos separados.

Como roteiro dos quase-experimentos, no início, a equipe de pesquisa se apresentou aos grupos e explicou os objetivos da pesquisa, os processos de coleta, o procedimento da análise de dados e como os resultados seriam importantes para dar base a hipóteses sobre o nível de participação ou refutá-las. Em seguida, os cases com as dinâmicas ou perguntas foram explicados e o cronômetro ligado para a marcação do tempo.

Os integrantes da equipe de pesquisa ficaram à disposição para tirar dúvidas iniciais, controlar o tempo, manter a dinâmica da deliberação e evitar dispersões. O término dos testes foi marcado pelo limite do tempo máximo estabelecido ou pela chegada ao consenso da equipe. Por fim, as assinaturas dos participantes foram 
recolhidas em listas de presença e termos de consentimento de uso de imagem, estes a pedido do Comitê de Ética na Pesquisa da Universidade.

Para a quantificação do nível de participação de cada participante, foram adotadas a contagem do tempo das falas de cada participante e a contagem do tempo de fala de todo o grupo. Para o cálculo do nível de participação, foi adaptado o cálculo do Coeficiente de Gini, nesse caso, para identificar o nível de distribuição igualitária dentro do grupo. Optou-se pela utilização desse coeficiente, pois há a possibilidade de se obter um cálculo mensurável de distribuição e também pela sua simplicidade na interpretação de suas informações.

O Coeficiente de Gini pode ser utilizado para calcular qualquer distribuição, embora seja comum o seu uso na mensuração da distribuição de renda, em que o valor máximo $(1,00)$ corresponde à concentração total da renda de uma comunidade política em uma única pessoa, e o valor mínimo $(0,00)$ corresponde à distribuição perfeita de renda, em que todos possuem igual capital.

Nesse experimento de participação de indivíduos em processos deliberativos, o Coeficiente de Gini - já transformado em valor percentual no seu resultado gráfico pela ferramenta utilizada - serviu para calcular o nível de participação na arena deliberativa, sendo o valor máximo (1,00 ou 100\%) correspondente à situação em que uma única pessoa concentra todas as falas e decisões do grupo e o valor mínimo $(0,00 \mathrm{ou} 0 \%)$, às situações em que houvesse uma distribuição plenamente igualitária do tempo de expressão de cada membro do grupo. Já os valores intermediários obtidos correspondem a processos em que algumas pessoas participam mais (concentram decisão) e outras participam menos.

Assim, o Coeficiente de Gini proporciona um escrutínio mais preciso sobre o elemento distributivo do fenômeno, nesse caso, sobre a distribuição de participação individual dentro de uma análise de grupo. A contagem em segundos do tempo de intervenção de cada participante pode não ser a representação perfeita da influência individual, mas é uma proxy mais robusta que modelos binários de "participou" versus "não participou".

O cálculo do Coeficiente de Gini foi realizado por meio da ferramenta Lorenz curve graphing tool \& Gini coefficient calculator (ROSENMAI, 2014). Mediante a análise das videogravações, calculou-se o tempo em segundos de fala dos participantes em cada quase-experimento, obtendo suas porcentagens de participação individuais ao dividir o tempo falado de cada participante pelo tempo da soma das falas de todos os participantes. O tempo total foi calculado com base na duração total do evento, diminuindo os minutos de falas sobrepostas e os momentos de silêncio. 
A observação do quase-experimento ocorreu in loco, durante a execução da simulação, e também a posteriori, por meio da análise dos vídeos para a verificação empírica do tempo de fala de cada participante.

A análise dos dados foi realizada por meio da transcrição dos vídeos e do cálculo de frequência das falas, sendo dispostos em uma tabela, e finalizada mediante a representação gráfica do nível de participação dos grupos em cada simulação por intermédio da Curva de Lorenz.

Os demais aspectos no âmbito da análise qualitativa, como as expressões faciais e corporais, bem como outros quesitos subjetivos, não foram avaliados, pois a intenção desse estudo foi apenas avaliar quantitativamente as intervenções individuais dos participantes no ambiente deliberativo, permitindo a testagem da hipótese da influência da urgência sobre o nível de participação.

Ao longo do quase-experimento vários esforços foram feitos para neutralizar fatores intervenientes. Os participantes e o formato da sala de simulação foram os mesmos, o nível de intervenção dos pesquisadores foi padronizado, o horário de aplicação dos semiexperimentos foi o mesmo, os cases de decisão foram muitos similares, a estratégia de contabilização e análise da participação foi a mesma. $\mathrm{O}$ único elemento que foi aplicado de forma heterogênea foi exatamente a variável independente (quantidade de tempo disponível para a deliberação, ou seja, urgência).

Há que se apontar limitações metodológicas do estudo, referente a outros elementos intervenientes ao nível de participação que não foram isolados. Um deles é o perfil do case deliberativo. Se fosse um case mais técnico, no qual valores e moral não estivessem em jogo, ou que exigisse conhecimento especializado por parte dos participantes, o nível de participação poderia ter sido outro. Outro fato é o número total de participantes, que ficou em 24 para o grupo de aposentados e 18 para o grupo de estudantes, números próximos, mas o efeito de tamanho de grupo pode inibir, estimular, constranger ou relaxar as intervenções individuais.

\section{APRESENTAÇÃO E DISCUSSÃO DOS RESULTADOS}

Ao analisar o fator urgência em uma arena deliberativa, procura-se descobrir se o comportamento do grupo sofre alguma influência sobre a percepção que os participantes possuem sobre a escassez do tempo. Os quase-experimentos relacionados a esse fator foram realizados com as turmas de alunos e aposentados na dinâmica de grupo Abrigo Subterrâneo. 
Os participantes deliberaram três vezes para chegar a um consenso: a primeira em um tempo com limitação de 20 minutos, a segunda com cinco minutos e a terceira com apenas dois minutos; em cada vez o case foi aplicado com personagens diferentes do case Abrigo Subterrâneo.

Na simulação com baixa urgência (20 minutos) com o grupo de aposentados, 16 dos 24 participantes se expressaram (66,66\%), em um tempo total em que as falas somadas resultaram em nove minutos e 40 segundos. Já o percentual de participantes ativos no grupo de alunos foi maior, pois, dos 16 participantes, 14 falaram (87,5\%), mas em tempo menor (quatro minutos e 46 segundos de falas aproveitáveis).

Na simulação com média urgência (cinco minutos), seis dos 22 aposentados falaram $(27,27 \%)$ com um tempo total de falas de um minuto e quarenta. Quanto aos alunos, essa simulação contou com a participação ativa de seis dos 15 presentes (40\%), e o tempo total de falas aproveitáveis foi de um minuto e seis segundos.

Na simulação com alta urgência (dois minutos), a participação de aposentados restringiu-se a apenas três pessoas do total de 22 participantes (13,63\%), com um tempo de 35 segundos de falas aproveitáveis. Já entre os alunos a participação foi maior, pois nove dos 15 alunos expressaram seus argumentos (60\%), com um total de 43 segundos.

Esses dados estão compilados nos quadros a seguir:

Quadro 1 - Síntese do tratamento de dados nas três simulações com aposentados

\begin{tabular}{|l|c|c|c|c|c|c|c|c|r|r|r|r|r|r|r|r|}
\hline $\begin{array}{c}\text { Parti- } \\
\text { cipan- } \\
\text { te }\end{array}$ & A & B & C & D & E & F & G & H & I & J & K & L & M & N & O & P \\
\hline $\begin{array}{l}\text { Segun- } \\
\text { dos de } \\
\text { fala }\end{array}$ & 171 & 86 & 65 & 63 & 43 & 43 & 30 & 23 & 17 & 14 & 8 & 6 & 4 & 3 & 2 & 2 \\
\hline $\begin{array}{l}\% \text { de } \\
\text { fala }\end{array}$ & 29,4 & 14,8 & 11,2 & 10,8 & 7,41 & 7,41 & 5,17 & 3,96 & 2,93 & 2,41 & 1,38 & 1,03 & 0,69 & 0,52 & 0,34 & 0,34 \\
\hline
\end{tabular}

Simulação 1 com aposentados

Baixa urgência: Tempo máximo concedido: 20 minutos. Tempo utilizado pelo grupo: 20

Tempo de falas aproveitáveis: $9 \min 40 \mathrm{~s}$

Número de participantes ativos: 16 (66,6\% dos 24 presentes)

\begin{tabular}{|c|r|r|r|r|r|r|}
\hline $\begin{array}{c}\text { Partici- } \\
\text { pante }\end{array}$ & A & B & C & D & E & \multicolumn{1}{c|}{ F } \\
\hline $\begin{array}{c}\text { Segundos } \\
\text { de fala }\end{array}$ & 33 & 32 & 17 & 11 & 5 & 2 \\
\hline$\%$ de fala & 33 & 32 & 17 & 11 & 5 & 2 \\
\hline
\end{tabular}




\section{Simulação 2 com aposentados}

Média urgência: Tempo máximo concedido: 5 minutos. Tempo utilizado pelo grupo: 5 minutos

Tempo de falas aproveitáveis: $1 \mathrm{~min} 40 \mathrm{~s}$

Número de participantes ativos: 6 (27,27\% dos 22 presentes)

\begin{tabular}{|c|r|r|r|}
\hline Participante & A & B & \multicolumn{1}{|c|}{ C } \\
\hline Segundos de fala & 19 & 11 & 14,28 \\
\hline \% de fala & 54,28 & 31,43 & \\
\hline
\end{tabular}

Simulação 3 com aposentados

Alta urgência: Tempo máximo concedido: 2 minutos. Tempo utilizado pelo grupo: 2 minutos

Tempo de falas aproveitáveis: 35 segundos

Número de participantes ativos: 3 (13,63\% dos 22 presentes)

Fonte: os autores.

Quadro 2 - Síntese do tratamento de dados nas três simulações com alunos

\begin{tabular}{|c|c|c|c|c|c|c|c|c|c|c|c|c|c|c|}
\hline $\begin{array}{c}\text { Parti- } \\
\text { cipan- } \\
\text { te }\end{array}$ & A & B & C & D & E & F & G & H & I & J & K & L & M & N \\
\hline $\begin{array}{c}\text { Segun- } \\
\text { dos de } \\
\text { fala }\end{array}$ & 62 & 49 & 36 & 30 & 24 & 24 & 19 & 16 & 10 & 8 & 5 & 3 & 2 & 1 \\
\hline $\begin{array}{c}\% \text { de } \\
\text { fala } \\
\text { aprox. }\end{array}$ & 21,4 & 16,9 & 12,4 & 10,4 & 8,3 & 8,3 & 6,57 & 5,54 & 3,46 & 2,77 & 1,73 & 1,04 & 0,7 & 0,34 \\
\hline
\end{tabular}

\section{Simulação 4 com alunos}

Baixa urgência: Tempo máximo concedido: 20 minutos. Tempo utilizado pelo grupo: 16 minutos e 20 segundos

Tempo de falas aproveitáveis: $4 \min 46 \mathrm{~s}$

Número de participantes ativos: 14 (87,5\% dos 16 presentes)

\begin{tabular}{|c|c|c|c|c|c|c|}
\hline Participante & A & B & C & D & E & F \\
\hline Segundos de fala & 15 & 15 & 14 & 11 & 6 & 5 \\
\hline \% de fala & 22,73 & 22,73 & 21,21 & 16,66 & 9,09 & 7,57 \\
\hline
\end{tabular}

\section{Simulação 5 com alunos}

Média urgência: Tempo máximo concedido: 5 minutos. Tempo utilizado pelo grupo: 4 minutos e 5 segundos

Tempo de falas aproveitáveis: $1 \mathrm{~min} 6 \mathrm{~s}$

Número de participantes ativos: 6 (40\% dos 15 presentes) 


\begin{tabular}{|c|r|r|r|r|r|r|r|r|c|}
\hline Participante & \multicolumn{1}{c|}{ A } & B & C & D & E & F & G & H & I \\
\hline Segundos de fala & 9 & 9 & 8 & 7 & 4 & 2 & 2 & 1 & 1 \\
\hline \% de fala aprox. & 20,93 & 20,93 & 18,6 & 16,28 & 9,3 & 4,65 & 4,65 & 2,32 & 2,32 \\
\hline
\end{tabular}

\section{Simulação 6 com alunos}

Alta urgência: Tempo máximo concedido: 2 minutos. Tempo utilizado pelo grupo: 2 minutos

Tempo de falas aproveitáveis: 43 segundos

Número de participantes ativos: 9 (50\% dos 18 participantes)

Fonte: os autores.

Figura 1 - Curva de Lorenz das seis simulações de deliberação
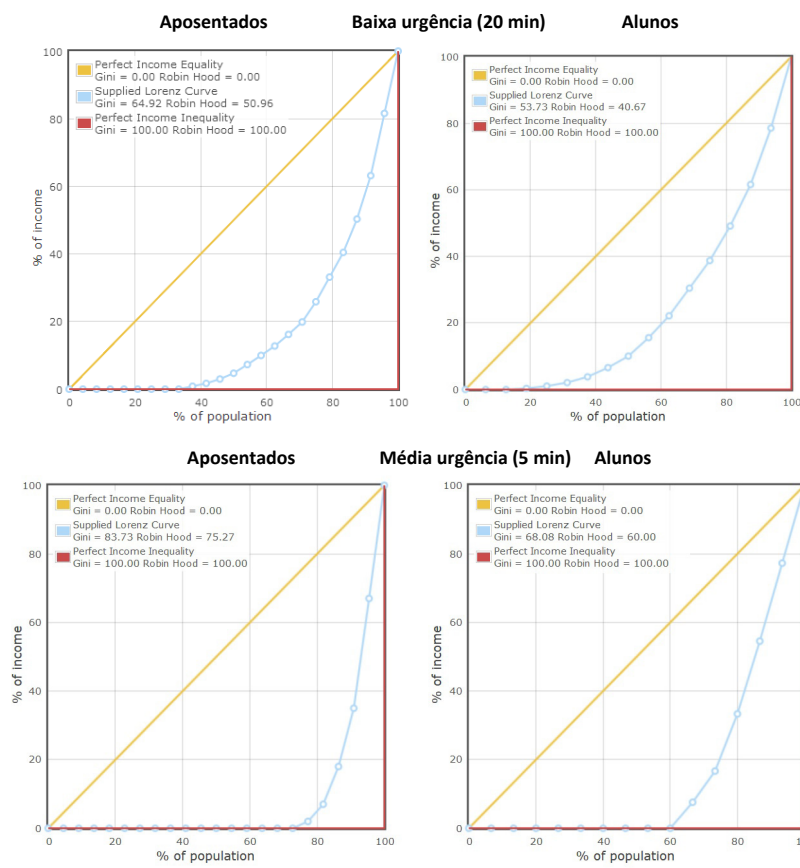

Média urgência (5 $\mathrm{min}$ ) Alunos
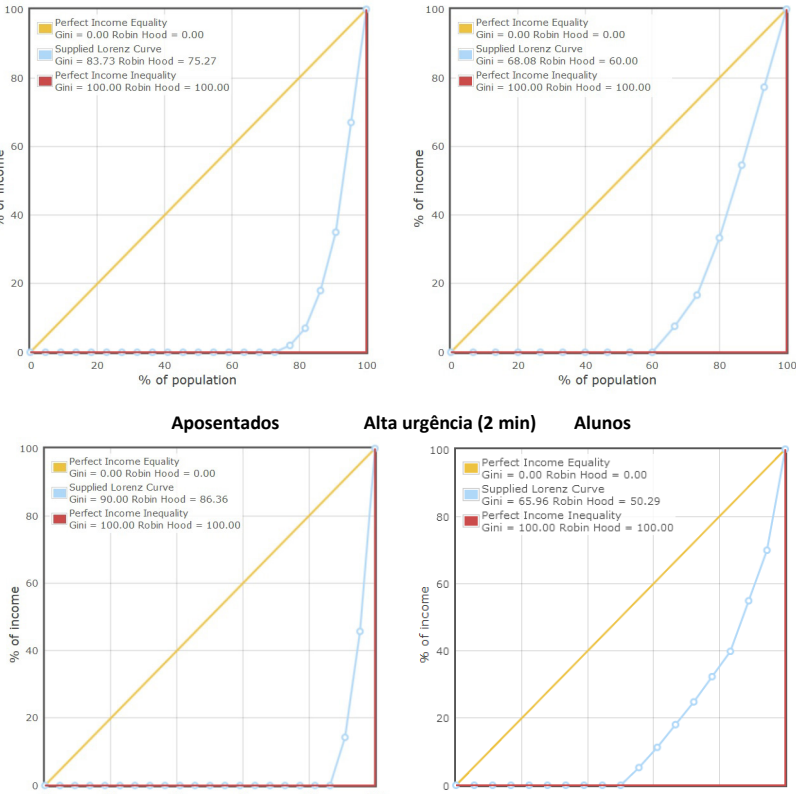

Fonte: os autores.

Nota: Gráfico gerado a partir de dados primários tratados pela ferramenta de gráfico da Curva de Lorenz e Calculadora do Coeficiente de Gini (RESENMAI, 2014). 
Como se pode perceber, as simulações tendem a corroborar a hipótese inicial de pesquisa. Em geral, quanto menor o tempo disponibilizado para a deliberação (maior urgência), menor foi o nível de participação dos sujeitos de pesquisa.

Nas simulações com os aposentados, o nível de participação diminui quando restringido o tempo de 20 minutos para cinco minutos, caindo de $66,6 \%$ de participação para 27,27\% e o Coeficiente de Gini mostrou maior concentração de participação (de 64,92 para 83,73). O nível de participação restringiu-se ainda mais na comparação da simulação de cinco minutos com a simulação de dois minutos, caindo de $27,27 \%$ de participantes ativos para apenas $13,63 \%$ e o Coeficiente de Gini agravou ainda mais a concentração (de 83,73 para 90 ).

Na simulação com os alunos as conclusões são muito parecidas. Os 87,5\% de participantes ativos na rodada de 20 minutos cairam para $40 \%$ de alunos participando ativamente nas discussões quando o tempo concedido foi de cinco minutos. $\mathrm{O}$ Coeficiente de Gini também mostrou maior concentração de participação, passando de 53,73 na rodada de 20 minutos, para 68,08 na rodada de cinco minutos. A única exceção para a corroboração da hipótese foi mostrada na comparação entre a rodada de cinco minutos com a rodada de dois minutos na simulação com os alunos. Contraintuitivamente, o nível de participação cresceu de 40 para $50 \%$ dos presentes, e o Coeficiente de Gini obteve ligeiro decréscimo de 68,08 para 65,96.

Se forem comparados os dois grupos, percebe-se que o grupo de alunos se demonstrou com maior nível de participação em todas as simulações se comparado com as simulações feitas com os aposentados. Em outras palavras, os alunos se apresentaram muito mais ativos e com distribuição mais igualitária das intervenções. Pesquisas adicionais são necessárias para corroborar ou refutar possíveis hipóteses da interferência da idade sobre a participação, resignação, proatividade e igualdade em processos deliberativos.

Uma possibilidade a ser considerada é a liderança informal que pode surgir no momento da deliberação, visto que é muito comum em uma sociedade ou em pequenos grupos o surgimento de lideranças (FAGUNDES, 2007). Inclusive, liderança que tem como papel facilitar para que o grupo consiga alcançar seus objetivos (TOLFO apud FAGUNDES, 2007). Secchi e Plebani (2006) propuseram que as arenas deliberativas possuem uma relação centro-periferia, ou seja, algumas pessoas assumem papéis mais relevantes e de centralidade organizacional, técnica ou decisória, obtendo um status relativamente maior que outros participantes do grupo (periferia), existindo um mínimo grau de hierarquia. 
Em um momento do quase-experimento realizado com o grupo de aposentados, por exemplo, notou-se o surgimento de um líder informal, que tentou organizar o processo deliberativo, tentando instituir uma ordem de falas dos participantes, evitando, assim, que houvessem discussões paralelas e corroborando uma deliberação mais rápida. Também sugere-se pesquisas adicionais para o tratamento da variável liderança informal (ex. existência ou não), e para tipos de lideranças informais (ex. centralizadora ou colaborativa) sobre o nível de participação em contextos deliberativas.

\section{CONCLUSÃO}

O senso comum indica que quanto maior a urgência, menor é a participação. Essa proposição também é tomada como verdadeira em diversos estudos teóricos de caráter dedutivo (SIMON, 1960; BEN-ZUR; BREZNITZ, 1981; PAPADAKIS; LIOUSKAS; CHAMBERS, 1998; IRVIN; STANSBURY, 2004; SECCHI; PLEBANI, 2006; GUNDERSEN; BOYER, 2012). O objetivo neste artigo foi analisar os efeitos da urgência sobre o nível de participação em processos deliberativos. Como pôde-se perceber, os quase-experimentos deste estudo tendem a corroborar a hipótese inicial de pesquisa. Em geral, quanto menor o tempo disponibilizado para a deliberação (maior urgência), menor é o nível de participação dos sujeitos de pesquisa.

Algumas limitações podem ser apontadas para a realização desse tipo de quase-experimento. As críticas recaem sobre a condição ceteris paribus, ou seja, que é impossível manter as condições ambientais de experimentação inalteradas. No entanto, limitações metodológicas sempre estarão presentes em estudos quaseexperimentais com seres humanos. Um dos elementos que podem enviesar é a motivação, bem como a condição emocional dos sujeitos de pesquisa. Não há indicações seguras para garantir que a motivação para a participação se mantenha inalterada na primeira, na segunda e na terceira simulação.

Também não é possível afirmar se o prévio conhecimento recíproco entre os sujeitos de pesquisa afetou ou não os resultados das simulações. Como eram alunos e aposentados que já se conheciam e mesmo de maneira informal já tinham identificado "lideranças informais", é natural imaginar que elementos como timidez, conflitos e coesões grupais tenham influenciado o processo de deliberação. 
Apesar dessas ressalvas, as teorias contingenciais são atentas a todos os fatores que interferem no nível de participação, como tecnicidade, custos de organização, nível de motivação, necessidade de sigilo, necessidade de legitimação, habilidade e maturidade dos participantes, necessidade de aprendizado e custos de oportunidade. Outro elemento é o modelo de tomada de decisão coletiva, que pode ser explorado também em contextos não deliberativos. A recomendação para o isolamento dessas interferências é a testagem em separado de cada uma das variáveis intervenientes, mantendo-se constantes as demais. Esse foi o procedimento adotado, sendo a urgência a única variável manipulada no caso.

Essa é uma agenda de pesquisas de longo prazo. Cada uma das variáveis intervenientes sobre o nível de participação merecem ser estudadas com métodos diferentes grupos sociais diferentes, e cada uma das variáveis estudadas separadamente. O acúmulo de estudos que, ao longo do tempo, corroborem, aperfeiçoem, refutem e consolidem os fatores intervenientes sobre o nível de participação é uma tarefa que exige foco e persistência por parte da comunidade epistêmica.

Considera-se que uma das grandes contribuições do presente estudo para essa agenda de pesquisa das teorias contingenciais é o seu perfil metodológico. A utilização do Coeficiente de Gini para calcular a concentração/distribuição das participações dos indivíduos se mostrou viável e eficaz. Sugere-se, portanto, a realização de mais pesquisas sobre o nível de participação utilizando-se o Coeficiente de Gini para o escrutínio de distribuição da participação em tomadas de decisão coletiva.

Como arenas deliberativas são utilizadas para a tomada de decisão em vários contextos na sociedade, com esta pesquisa, fez-se uma tentativa de medir alguns dos fatores que podem influenciar esse processo. Cabe aos pesquisadores da área, assim, analisar esses dados e confrontá-los ou aprová-los com novas pesquisas, a fim de revelar até que ponto o nível de participação é influenciado por determinadas características do ambiente e de seus participantes, para que a tomada de decisão coletiva possa ser utilizada de maneira mais benéfica às organizações públicas e privadas.

Nota explicativa

${ }^{1}$ Ao longo das simulações, algumas pessoas deixaram o ambiente de simulação por motivos pessoais. No grupo de aposentados, o total de participantes variou entre 22 e 24 e no grupo de alunos, variou entre 15 e 18. Nos cálculos do Coeficiente de Gini e da Curva de Lorenz foram consideradas essas variações. 


\section{REFERÊNCIAS}

AVRITZER, L. Teoria democrática e deliberação pública. Lua Nova, n. 49, p. $25-$ 46, 2000 .

BARISIONE, M. Framing a Deliberation: Deliberative Democracy and the Challenge of Framing Processes. Journal of Public Deliberation, v. 8, 2012.

BARTOL, K. M.; MARTIN, D. C. Management. New York: McGraw-Hill, 1991.

BEN-ZUR H.; BREZNITZ, S. J. The effect of time pressure of risky choice behaviour. Acta Psychologica, v. 47, p. 89-104, 1981.

BOHMAN, J. Public deliberation. Cambridge: MIT Press, 1996.

CALSYDE, P. P. F. M. V. de; KEREN, G.; ZEELENBERG, M. Decision time as information in judgment and choice. Organizational behavior and Human Decision Process, v. 125, p. 113-122, 2014.

COASE, R. The Problem of Social Cost. Journal of Law and Economics, v. 3, i. 1,1960 .

CURINI, L. Vox populi - Vox Dei? (alcuni) limiti e (alcuni) paradossi della pratica deliberativa. Rivista Italiana di Scienze Politiche, v. 2, p. 231-257, 2006.

ELIAS, N. Sobre o tempo. Rio de Janeiro: J. Zahar, 1998.

EMMENDOERFER, M. L. Controle do tempo: a percepção dos atores gerenciais da Usiminas S.A. 2004. Dissertação (Mestrado em Administração)Universidade Federal de Minas Gerais, 2004.

FAGUNDES, P. M. Desenvolvimento de competências coletivas de liderança e de gestão: uma compreensão sistêmico-complexa sobre o processo de organização grupal. 2007. Tese (Doutorado em Psicologia)Pontifícia Universidade Católica do Rio Grande do Sul, Porto Alegre, 2007.

Disponível em: < http://tede.pucrs.br/tde_busca/arquivo.php?codArquivo $=1334>$. Acesso em: 21 jul. 2015.

FARRAR, C. et al. Disaggregating deliberation's effects: an experiment within a deliberative poll. British Journal of Political Science, 2010.

FIEDLER, F. E. A Theory of Leadership Effectiveness. New York: McGrawHill, 1967. 
GIL, A. C. Métodos e técnicas de pesquisa social. 6. ed. São Paulo: Atlas, 2011. GRANDORI, A. A prescriptive contingency view of organizational decisionmaking. Administrative Science Quarterly, v. 29, p. 192-209, 1984.

GUNDERSEN, A. G.; BOYER, D. Just-in-time exploratory public discussion. Journal of Public Deliberation, v. 8, i. 1, 2012.

HERSEY, P.; BLANCHARD, K. H. Management of organizational behavior: utilizing human resources. Englewood Cliffs: Prentice Hall, 1993.

IRVIN, R. A.; STANSBURY, J. Participation in Decision Making: Is It Worth the Effort? Public Administration Review, v. 4, i. 1, p. 55-65, Jan./Feb. 2004.

KERLINGER, F. N. Metodologia da pesquisa em ciências sociais: um tratamento conceitual. São Paulo: EPU, 1980.

NAGEL, J. H. Participation. Englewood Cliffs: Prentice-Hall, 1987.

PAPADAKIS, V. M., LIOUSKAS, S.; CHAMBERS, D. Strategic decision-making processes: the role of management and context. Strategic Management Journal, v. 19, p. 115-147, 1998.

PAYNE, J. W.; BETTMAN, J. R.; LUCE, M. F. When time is money: decision behavior under opportunity-cost time pressure. Organizational Behavior and Human Decision Process, v. 66, i. 2, p. 131-152, 1996.

ROSENMAI, P. Lorenze curve graphing tool and Gini coefficient calculator. 2012. Disponível em: < http://www.peterrosenmai.com/lorenz-curvegraphing-tool-and-gini-coefficient-calculator >. Acesso em: 07 jul. 2014.

SECCHI, L.; PLEBANI, F. The level of participation in deliberative arenas. In: ENANPAD, 30., 2006, Salvador. Anais... Salvador, 2006.

SELLTIZ, C.; WRIGHTSMAN, L. S.; COOK, S. W. Métodos de pesquisa nas relações sociais. 2. ed. São Paulo: EPU, 1987.

SIMON, H. A. The new science of management decision. 3. ed. Englewood Cliffs: Prentice-Hall, 1977.

STEINER, I. D. Group process and productivity. New York: Academic Press, 1972.

VROOM, V. H.; JAGO, A. G. The new leadership: managing participation in organizations. Englewood Cliffs: Prentice Hall, 1988. 


\section{COMO CITAR ESTE ARTIGO:}

SECCHI, Leonardo; FEIJÓ, Juliana Kulpa. Efeitos da urgência sobre o nível de participação em processos deliberativos. RACE, Revista de Administração, Contabilidade e Economia, Joaçaba: Ed. Unoesc, v. 14, n. 3, p. 905-924, set./ dez. 2015. Disponível em: < http://editora.unoesc.edu.br/index.php/race>. Acesso em: dia/mês/ano.

Secchi, L., \& Feijó, J. K. (2015). Efeitos da urgência sobre o nível de participação em processos deliberativos. RACE, Revista de Administração, Contabilidade e Economia, 14 (3), 905-924. Recuperado em dia/mês/ano, de http://editora.unoesc.edu.br/ index.php/race 
